\title{
L. Contributions to the theory of solutions
}

\section{Vegard}

To cite this article: L. Vegard (1907) L. Contributions to the theory of solutions, Philosophical Magazine Series 6, 13:77, 589-613, DOI: 10.1080/14786440709463636

To link to this article: http://dx.doi.org/10.1080/14786440709463636

册 Published online: 16 Apr 2009.

Submit your article to this journal $\pi$

Џ Article views: 4

Q View related articles $₫$ 
L. Contritutions to the Theory of Solutions. By L, Vegard (Physical Institute, The Úniversity, Kristiania)*.

$\S 1$.

On the Change of Concentration in a Binary Solution, which is in a field of force where the force is proportional to the ponderable mass.

$\mathbf{T}$ the thermodynamic treatment of mixtures of substances dissolved in each other, it is always understood that the effect of Gravity need not be considered. In other words, a solution is supposed homogeneous, provided that equilibrium has set in. It follows, then, that the functions become homogeneous with regard to the components of the mixture.

If, however, the solution is submitted to the infiuence of a force that is proportional to the ponderable mass of the individual components-exposed to the influence of Gravity for instance-the homogeneity will be lost, since the pressure varies from place to place in the fluid. Besides, it is a priori probable that the proportions of mixture also change within the solution, when the latter is in equilibrium. For if there were no inclination on the part of the substances to dissolve in each other, Gravity would cause the components to accumulate on the top of each other, each according to its own specific density. This effect of Gravity is now counteracted by the inclination towards solution; an equilibrium must ensue, in which the proportions of mixture change from place to place, so that the Centre of Gravity of the whole lies lower than if the system were homogeneous, but higher than if the substances had not dissolved in each other. In a solution exposed to the influence of Gravity, the thermodynamic functions respecting the components will be homogeneous no longer. In the neighbourhood of any point they can, however, be considered homogeneous.

Let us suppose that we have, in the following, a binary solution, that is to say, that we only have two components in the fluid system. Let us further suppose that the field to which the systera is exposed is dependent on a potential $U$, which may be given as a function of coordinates in a rectangular coordinate system. $U$ as well as its first partial derivatives are considered to be continuous in the space occupied by the system.

Let the two components have molecular masses $M_{1}$ and $M_{2}$.

* Communicated by the Author. Placed before Kristiania Videnskubsselskab on the 12th of October, 1906. 
If in a small volume element of the solution there are $n_{1}$ molecules of the first and $n_{2}$ of the second component, the concentration will be defined by the equation

$$
c=\frac{\mathrm{M}_{2} n_{2}}{\mathrm{M}_{1}} .
$$

The task we now set before us, is to find $c$ as a function of the coordinates when equilibrium has set in, and the temperature is the same all over the system.

To keep the system in equilibrium, two conditions must be present-one mechanical and one thermodynamic. If the pressure at a point in the fluid is given by $p$, it gives the following mechanical condition for equilibrium :

$$
d p=-\rho\left(\frac{\partial \mathrm{U}}{\partial x} d z+\frac{\partial \mathrm{U}}{\partial y} d y+\frac{\partial \mathrm{U}}{\partial z} d z\right)
$$

$\rho$ is the density of the solution at the point considered. To maintain equilibrium it is absolutely necessary for $p$ to be a function of the coordinates, $i$. $e$., $d p$ must be an exact differential. Consequently:

$$
\begin{gathered}
\frac{\partial\left(\rho \frac{\partial \mathrm{U}}{\partial x}\right)}{\partial y}=\frac{\partial\left(\rho \frac{\partial U}{\partial y}\right)}{\partial x} \\
\frac{\partial\left(\rho \frac{\partial U}{\partial x}\right)}{\partial z}=\frac{\partial\left(\rho \frac{\partial U}{d z}\right)}{\partial x} .
\end{gathered}
$$

On differentiation and rearrangement;

$$
\frac{\frac{\partial \rho}{\partial x}}{\frac{\partial U}{\partial x}}=\frac{\frac{\partial \rho}{\partial y}}{\frac{\partial y}{\partial y}}=\frac{\frac{\partial \rho}{\partial \bar{U}}}{\partial z}=\frac{\frac{\partial \rho}{\partial n}}{-\bar{K}}
$$

where $\mathrm{K}$ denotes the resultant of the force intensity at the point, and $\frac{\partial \rho}{\partial n}$ the changs of density per unit of length in the direction of the force.

As the density is a function of the coordinates,

$$
d \rho=\frac{\partial \rho}{\partial x} d x+\frac{\partial \rho}{\partial y} d y+\frac{\partial \rho}{\partial z} d z
$$

or by use of the above equation

$$
d \rho=-\frac{\partial \rho}{\partial n} \frac{1}{\tilde{\mathrm{K}}} d \mathrm{U} .
$$


Furthermore, the concentration can be stated as a function of pressure, temperature, and density, If the temperature is always kept constant,

$$
d c=\frac{\partial c}{\partial \rho} d \rho-\frac{\partial c}{\partial p^{\prime}} d p
$$

If the values of $d \rho$ and $d p$ are substituted,

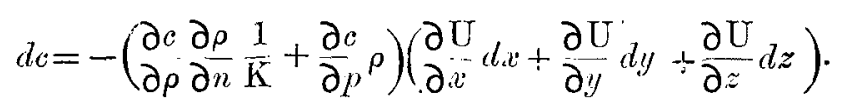

We must also be able to express it thus :

$$
d c=\frac{\partial c}{\partial i c} d z+\frac{\partial c}{\partial y} d y+\frac{\partial c}{\partial z} d z . \quad \text {. . . . }
$$

And since both the terms for $d c$ must be identical, and $x, y, z$ are independent variables,

$$
\frac{\frac{\partial c}{\partial x}}{\frac{\partial \vec{U}}{\partial x}}=\frac{\frac{\partial c}{\partial u}}{\frac{\partial U}{\partial y}}=\frac{\frac{\partial c}{\partial z}}{\partial \vec{U}}=\frac{\frac{\partial c}{\partial n}}{\partial \vec{z}} .
$$

If these equations are inserted in $(2 a)$

$$
d c=-\frac{1}{\mathrm{~K}} \frac{\partial}{\partial} \frac{\partial}{\partial} d \mathrm{U} . \quad . \quad . \quad .
$$

From this equation it appears that $d_{c}=0$ when $d \mathrm{U}=0$. From this we conclude, that in the state of equilibrium the concentration must be constant along a potential surface and must have the greatest slope in the direction of the force. $\mathrm{U}$ and $\mathrm{K}$ are given quantities. The only thing we now have to determine is $\frac{\partial c}{\partial n}$; or, as we might call it, the concentration gradient. This must be determined by help of the thermodynamic equilibrium condition, which we shall briefly mention.

If at constant temperature a system suffers a change from a state 0 to a state 1 , the amount of heat that the system disengages during the change can, as is well known, be expressed as follows:

$$
\mathrm{Q}=\mathrm{T}\left(\mathrm{S}_{0}-\mathrm{S}_{1}\right)+\mathrm{TP} \text {. }
$$

Here $T$ means the absolute temperature, $S$ the entropy, $T\left(S_{0}-S_{1}\right)$ the amount of heat that the systems would have disengaged if the process had been carried out reversibly. 
According to the second law of thermodynamics $\mathrm{P}$ is a quantity that, for all processes which can arise in the system of their own accord, can never be negative.

If our system is considered in the neighbourhood of the point of equilibrium the vis viva can be left out of consideration, and in accordance with the First Law of Thermodynamies

$$
\mathrm{Q}=\mathrm{I}_{0}-\mathrm{I}_{1}+\mathrm{A},
$$

the heat being measured in work units. $I$ is the internal energy, and A the external work done by the forces of the system.

By a combination of both these equations:

$$
\mathrm{TP}=\left(\mathrm{I}_{0}-\mathrm{TS}_{0}\right)-\left(\mathrm{I}_{1}-\mathrm{TS}_{1}\right)+\mathrm{A} .
$$

Here I and S are functions of the variables of the system. We put

$$
\mathrm{I}-\mathrm{TS}=\psi,
$$

where $\psi$ is the so-called thermodynamic potential. If the system suffers an infinitely slight change :

$$
\mathrm{T} \delta \mathrm{P}=-\delta \psi+\delta \mathrm{A} .
$$

The condition which is necessary, in order that a system left to itself at constant temperature, shall be in equilibrium, is that $\delta \mathrm{P}=0$ for every small change that the system may suffer*. If the system depends on $r$ independent variables $\alpha_{1}, \alpha_{2} \ldots \alpha_{r}$, the system can suffer $p$ independent variations. The equilibrium condition may therefore be expressed as follows:

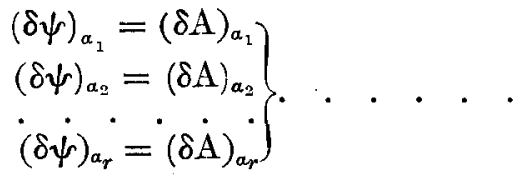

Here $(\delta \psi)_{a_{r}}$ and $(\delta \mathrm{A})_{a_{r}}$ mean the changes respectively of $\psi$ and $A$ for the same variation of $\alpha_{r}$, when the other variables are kept invariable.

The change that must come into consideration here is a relative displacement of both components in a volume element. This variation will generally cause a small volume change. The pressure on the element will, however, not be changed by it. If the external pressure is kept constant during the

* Duhem, Mécanique Chimique, tome i. livre i. p. 89. 
change, the exterior work will consist of two parts, and we have

$$
\delta \mathrm{A}=-p \delta v+\delta a,
$$

$p$ is the pressure, $v$ the volume, and $\delta a$ the work done by the force in the field, on account of the variation. The equilibrium condition will in our case then be

and as $p$ is constant,

$$
\delta \psi+p \delta v=\delta a ;
$$

$$
\delta(\dot{\psi}+p v)=\delta a,
$$

$(\psi+p v)$ is the exterior thermodynamic potential or the thermodynamic potential under constant pressure. If this function is indicated by $\omega$, we get

$$
\delta \omega=\delta a . . . . . . .
$$

We will now consider a small volume element $d x, d y, d z$, containing $n_{1}$ molecules of the first component, and $n_{2}$ molecules of the second component. We select the $\mathrm{X}$ axis parallel to the force at the point considered. Let ns suppose that the thermodynamic potential for the element-neglecting quantities of a higher order-has the same value as it would have had if the system had been homogeneous, and pressure and concentration had had the same value as is to be found in the centre of the element. $\omega$ then becomes for the element a homogeneous function of the first order; and according to a well-known theorem of Euler

$$
\omega\left(\mathrm{M}_{1} n_{1}, \mathrm{M}_{2} n_{2}, p, \mathrm{~T}\right)=\mathrm{M}_{1} n_{1} \frac{\partial \omega}{\partial\left(\mathrm{M}_{1} n_{1}\right)}+\mathrm{M}_{2} n_{2} \frac{\partial \omega}{\partial\left(\mathrm{M}_{2} n_{2}\right)} .
$$

The two partial derivatives are again homogeneous functions of the 0th degree of the components. We put

$$
\begin{gathered}
\frac{\partial \omega}{\partial\left(\bar{M}_{1} n_{1}\right)}=f_{1}(c, p, \mathrm{~T}) \\
\frac{\partial \omega}{\partial\left(\mathrm{M}_{2} n_{2}\right)}=f_{2}(c, p, \mathrm{~T}) .
\end{gathered}
$$

If we once more make use of Euler's theorem the result will be

$$
\mathrm{M}_{1} n_{1} \frac{\partial^{2} \omega}{\partial\left(\mathrm{M}_{1} n_{1}\right)^{2}}+\mathrm{M}_{2} n_{2} \frac{\partial^{2} \omega}{\partial\left(\mathrm{M}_{1} n_{1}\right) \partial\left(\mathrm{M}_{2} n_{2}\right)}=0
$$

Phil. Mag. S. 6. Vol. 13. No. 77. May 1907. 
If $f_{1}(c, p, \mathrm{~T})$ and $f_{2}(c, p, \mathrm{~T})$ are introduced, we get the following very important combination:

$$
\frac{\partial f_{1}(c, p, \mathrm{~T})}{\partial c}+c \frac{\partial f_{2}(c, p, \mathrm{~T})}{\partial c}=0^{*} . \quad . \quad .
$$

If $f_{1}^{\prime}$ and $f_{2}$ are introduced in equation $(4 a)_{2}^{*}$ we get

$$
\omega=\mathrm{M}_{1} n_{1} f_{1}(e, p, \mathrm{~T})+\mathrm{M}_{2} n_{2} f_{2}(c, p, \mathrm{~T}) . .
$$

If we now add to $x$ a small quantity $\Delta x=d x$ we get a new element of the same capacity as the original (see fig. 1).

Fig. 1.

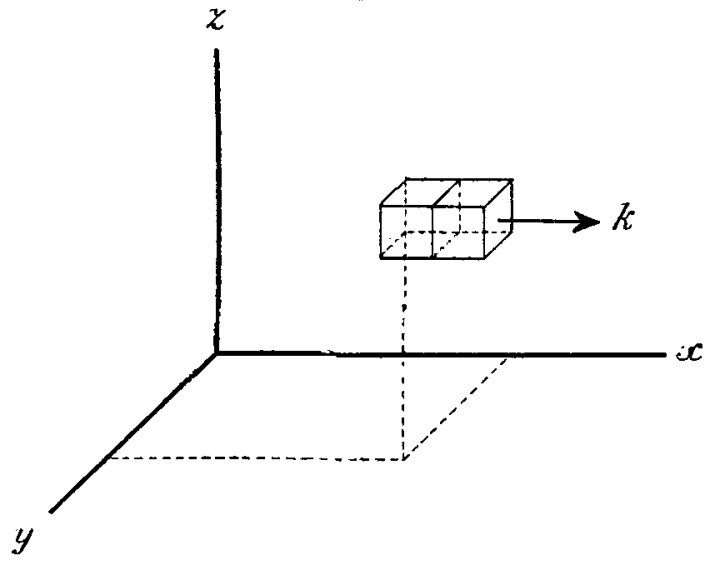

On the average the pressure will be a different one in this element, and besides the mixture proportion will also be different. Supposing the numbers of molecules of the new element were $n_{1}+d n_{1}$ and $n_{2}+d n_{2}$, this will involve the following change in the concentration

$$
d c=c\left(-\frac{d n_{1}}{n_{1}}+\frac{d n_{2}}{n_{2}}\right) . . . . .
$$

If we leave out quantities of the second and higher orders, we get for the thermodynamic potential of the second element

$$
\begin{aligned}
\omega^{\prime}=\omega+\mathrm{M}_{1} f_{1} d n_{1}+\mathrm{M}_{2} f_{2} d n_{2}+ & \mathrm{M}_{1} n_{1}\left(\frac{\partial f_{1}}{\partial c}+c \frac{\partial f_{2}}{\partial c}\right) d c \\
& +\mathrm{M}_{1} n_{1}\left(\frac{\partial f_{1}}{\partial p}+c \frac{\partial f_{2}}{\partial p}\right) d p .
\end{aligned}
$$

* Duhem, loc. cit. tome iii. livre vi. p. 5 . 
Here $d n_{1}, d n_{2}, d c, d p$, mean the average changes of the four quantities $n_{1}, n_{2}, c, p$ from one element to the other; or rather the changes which these quantities undergo when we go from the centre of the first element to the centre of the second. Then we can write

$$
\begin{aligned}
d n_{1} & =\frac{\partial n_{1}}{\partial x} d x, & d n_{2} & =\frac{\partial n_{2}}{\partial x} d x, \\
d l & =\frac{\partial c}{\partial x} d x, & d p & =\frac{\partial p}{\partial x} d x,
\end{aligned}
$$

and when we take equations (1) and (5) into consideration:

$$
\boldsymbol{\omega}^{\prime}=\boldsymbol{\omega}+\left[\mathrm{M}_{1} f_{1} \frac{\partial n_{1}}{d x}+\mathrm{M}_{2} f_{2} \frac{\partial n_{2}}{\partial x}+\mathrm{M}_{1} n_{1}\left(\frac{\partial f_{1}}{\partial p}+c \frac{\partial f_{2}}{\partial p}\right) \rho \mathrm{K}\right] d x
$$

It must now be noticed that $n_{1}$ and $n_{2}$ do not change independently of each other; for there must always be sufficient substance of both kinds in each element to keep the volume element full of fluid.

Supposing that the molecuiar volumes of the components are indicated at the concentration $c$ by $v_{1}$ and $v_{2}$ respectively, the volume of the first element will then be

$$
d w \cdot d y \cdot d z=v_{1} n_{1}+v_{2} n_{2} .
$$

In the other the concentration is different; and the pressure also. Let us, however, suppose that the fluid is incompressible, and that we leave out of consideration the change of volume with pressure. The molecular volumes of both components of the second element will then be

$$
\left(v_{1}+\frac{\partial v_{1}}{\partial c} d c\right) \text { and }\left(v_{2}+\frac{\partial v_{2}}{\partial c} d c\right)
$$

and the volume of the second element

$$
\begin{aligned}
\left(v_{1}+\frac{\partial v_{1}}{\partial c} d c\right)\left(n_{1}+d n_{1}\right) & +\left(v_{2}+\frac{\partial v_{2}}{\partial c} d c\right)\left(n_{2}+d n_{2}\right) \\
& =d x \cdot d y \cdot d z=v_{1} n_{1}+v_{2} n_{2} .
\end{aligned}
$$

For brevity's sake let us write

$$
\begin{aligned}
& k_{1}=v_{1}-\left(\frac{\partial v_{1}}{\partial c}+\frac{\mathbf{M}_{1}}{\mathbf{M}_{2}} c \frac{\partial r_{2}}{\partial c}\right) c \\
& k_{2}=v_{2}+\left(\frac{\partial v_{1}}{\partial c}+\frac{M_{1}}{\bar{M}_{2}} c \frac{\partial v_{2}}{\partial c}\right) \frac{M_{2}}{\bar{M}_{1}} .
\end{aligned}
$$


Then we get

$$
k_{1} d n_{1}+k_{2} d n_{2}=0 .
$$

Or as $d n_{1}$ and $d n_{2}$ are variations corresponding to the same variation $d x$ :

$$
k_{1} \frac{\partial n_{1}}{\partial x}+k_{2} \frac{\partial n_{2}}{\partial x}=0 . . . . .
$$

If now $\frac{\partial n_{1}}{\partial x}$ is eliminated between equations $(7 a)$ and $(8 a)$ we get

$$
\boldsymbol{\omega}^{\prime}=\boldsymbol{\omega}+\left[\left(\mathrm{M}_{1} f_{1}^{\prime}-k_{k_{2}}^{k_{1}} \mathrm{M}_{2} f_{2}\right) \frac{\partial{ }^{\prime}{ }^{\prime} x}{\partial x}+\mathrm{M}_{1} n_{1}\left(\frac{\partial f_{1}}{\partial p}+c \frac{\partial f_{2}}{\partial p}\right) \rho \mathrm{K}\right] d x .
$$

The thermodynamic potential for both elements taken together will be

$$
\omega^{\prime}+\omega=2 \omega+\left[\left(\mathrm{M}_{1} f_{1}-\frac{k_{1}}{k_{2}} \mathrm{M}_{2} f_{2}\right) \frac{\partial n}{\partial x}+\mathrm{M}_{1} n_{1}\left(\frac{\partial f_{1}}{\partial p}+c \frac{\partial f_{2}}{\partial p}\right) \rho \mathrm{K}\right] d x .
$$

In urder to find the condition for equilibrium, we must carry out a small variation, which consists in the masses $\mathrm{M}_{1} \in n_{1}$ and $\mathrm{M}_{2} \in n_{2}$ being carried from the first to the second element. These variations $\epsilon n_{1}$ and $\epsilon n_{2}$ are not, however, independent of each other but are connected by the equation

$$
v_{1} \epsilon n_{1}+v_{2} \epsilon n_{2}=0 . \text {. . . . . }
$$

This equation $(8 b)$ can with complete mathematical exactness only be applied on the supposition that the molecular volumes do not vary with the concentration. As, however, we shall see later on, the result must also, on account of the continuity of nature, approximately be applicable to such solutions for which the concentration is quite small ; and this is really the case with most solutions. And furthermore, the actual change of concentration in the state of equilibrium is a very small quantity.

The thermodynamic potential after the variation of our two elements, can now be found by calculating the potential of each element separately.

The thermodynamic potential after the variation will then be for the first element,

$$
\omega_{1}=\omega-\left(M f_{1}-\frac{v_{1}}{v_{2}} \mathrm{M}_{2} f_{2}\right) \epsilon n_{1}
$$


for the second,

$$
\begin{aligned}
\omega_{1}^{\prime} & =\omega+\left[\left(\mathrm{M}_{1} f_{1}-\frac{k_{1}}{k_{2}} \mathrm{M}_{2} f_{2}\right) \frac{\partial n_{1}}{\partial \cdot v^{\prime}}+\mathrm{M}_{1} n_{1}\left(\frac{\partial f_{1}}{\partial p}+c \frac{\partial f_{2}}{\partial p}\right) \rho \mathrm{K}\right] d v \\
& +\left(\mathrm{M}_{1} f_{1}-\frac{v_{1}}{v_{2}} \mathrm{M}_{2} f_{2}\right) \epsilon n_{1}+\left[\left(\mathrm{M}_{1} \frac{\partial f_{1}}{\partial c}-\frac{k_{1}}{k_{2}} \mathrm{M}_{2} \frac{\partial f_{2}}{\partial c}\right) \frac{\partial n}{\partial x} \epsilon c\right. \\
& \left.+\rho . \mathrm{K} \cdot\left(\mathrm{M}_{1} \frac{\partial f_{1}}{\partial p}-\frac{v_{1}}{v_{2}} \mathrm{M}_{2} \frac{\partial f_{2}}{\partial p}\right) \epsilon n_{1}+\mathrm{M}_{1} n_{1} \cdot \rho \cdot \mathrm{K}\left(\frac{\partial^{2} f_{1}}{\partial e \partial p}+c \frac{\partial^{2} f_{2}}{\partial e \partial p}\right) \epsilon c\right] d x .
\end{aligned}
$$

On a superficial view, one would have thought that also $\rho$, as a function of the concentration, would bave varied here. We must, however, remember that $\rho$ is the average density between the centres of the elements; and by this relative exchange of masses nothing will be carried away from the whole double element. The average density keeps its value unaltered, and change of pressure between the centres is not influenced by the variation.

The above term for $\omega_{1}^{\prime}$ can be considerably simplified, since

$$
\frac{\partial^{2} f_{1}}{\partial c \partial p}+\frac{\partial^{2} f_{2}}{\partial c \partial p} c=\frac{\partial}{\partial p}\left(\frac{\partial f_{1}}{\partial c}+c \frac{\partial f_{2}}{\partial c}\right)=0 .
$$

As will be seen later,

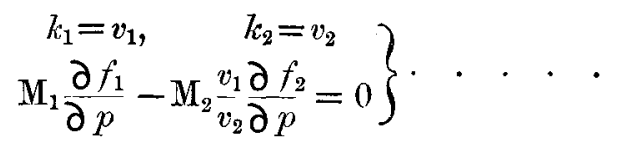

The term for $\omega_{1}^{\prime}$ will then appear as follows :

$$
\omega_{i}^{\prime}=\omega^{\prime}+\left(\mathrm{M}_{1} f_{1}-\frac{v_{1}}{v_{2}} \mathrm{M}_{2} f_{2}\right) \epsilon n_{1}+\left(\mathrm{M}_{1} \frac{\partial f_{1}}{\partial c}-\frac{v_{1}}{v_{2}} \frac{\partial f_{2}}{\partial c} \mathrm{M}_{2}\right) \frac{\partial n_{1}}{\partial x} \epsilon e d x .
$$

By the aid of equations (5), (8a), (8b), and (9) we get:

$$
\begin{aligned}
& d c=-c\left(\frac{1}{n_{1}}-\frac{v_{1}}{v_{2}} \frac{1}{n_{2}}\right) d n_{1} \\
& \epsilon c=-c\left(\frac{1}{n_{1}}-\frac{v_{1}}{v_{2}} \frac{1}{n_{2}}\right) \epsilon n_{1},
\end{aligned}
$$

and consequently

$$
\frac{\partial n_{1}}{\partial x} \epsilon c=\frac{\partial c}{\partial x} \epsilon n_{1} .
$$

If this is introduced into the equation for $\omega_{1}{ }^{\prime}$, we get at last for the whole variation of the thermodynamic potential :

$$
\delta \omega=\left(\omega_{1}+\omega_{1}^{\prime}\right)-\left(\omega+\omega^{\prime}\right)=\left(\mathrm{M}_{1} \frac{\partial f_{1}}{\partial c}-\frac{v_{1}}{v_{2}} \mathbf{M}_{2} \frac{\partial f_{2}^{\prime}}{\partial c}\right) \frac{\partial c}{\partial x} \epsilon n_{1} d s .
$$


We have still to find the work done by the force during the variation. On the average the masses have been moved a space $d x$, in the direction of the force, and accordingly

$$
\delta a=\mathrm{K}\left(\mathrm{M}_{1} \epsilon n_{1}+\mathrm{M}_{2} \epsilon n_{2}\right) d x ;
$$

or by the aid of equation $(8 b)$ :

$$
\delta a=\mathrm{K}\left(\mathrm{M}_{1}-\frac{v_{1}}{v_{2}} \mathrm{M}_{2}\right) \epsilon n_{1} d x . \quad . \quad .
$$

If the values found for $\delta \omega$ and $\delta a$ (equations $(10 a)$ and $(10 b)$ ) are introduced into equation (3), we get as the condition for equilibrium :

$$
\left(\frac{\mathrm{M}_{1}}{v_{1}} \frac{\partial f_{1}(c, p, \mathrm{~T})}{\partial c}-\frac{\mathrm{M}_{2}}{v_{2}} \frac{\partial f_{2}(c, p, \mathrm{~T})}{\partial c}\right) \frac{\partial c}{\partial x}=\mathrm{K}\left(\frac{\mathrm{M}_{1}}{v_{1}}-\frac{\mathrm{M}_{2}}{v_{2}}\right) .
$$

If we now eliminate $\frac{\partial f_{2}}{\partial e}$ by the aid of equation (5) and write-in view of equation (2) $-n$ instead of $x$,

$$
\frac{\partial f_{1}(c, p, \mathrm{~T})}{\partial c}\left(\frac{\mathrm{M}_{1}}{v_{1}}+\frac{1}{c} \frac{\mathrm{M}_{2}}{v_{2}}\right) \frac{\partial c}{\partial n}=\mathrm{K}\left(\begin{array}{c}
\mathrm{M}_{1} \\
v_{1}
\end{array}-\frac{\mathrm{M}_{2}}{v_{2}}\right) \text {. }
$$

From this equation it appears that when the solution is in equilibrium, the concentration gradient is at every point proportional to the force.

In equation (11a) we will try to introduce quantities that are more spontaneously accessible, and begin by finding other values for the ratios $\frac{\mathrm{M}_{1}}{v_{1}}$ and $\frac{\mathrm{M}_{2}}{v_{2}}$. These quantities mean the molecular masses divided by the molecular volumes. To make clearer the meaning of these quantities, we may suppose that we bave a very big quantity of the solution at the concentration $c$. If to the fluid is added a mass $\mathbf{M}_{1}$ of the first or $\mathrm{M}_{2}$ of the second component, the volume will be increased by the quantities $v_{1}$ and $v_{2}$ respectively.

Instead of an infinite, we consider a finite quantity and add infinitely small masses $\mathbf{M}_{1} d n_{1}$ and $\mathbf{M}_{2} d n_{2}$ to the solution. By doing that the volumes will be increased by $d v_{1}$ and $d v_{2}$; and then

$$
\frac{\mathrm{M}_{1}}{v_{1}}=\frac{\mathrm{M}_{1} d n_{1}}{d v_{1}} \text { and } \frac{\mathrm{M}_{2}}{v_{3}}=\frac{\mathrm{M}_{2} d n_{2}}{d v_{2}} \text {. }
$$

If the whole volume of the solution is $\mathrm{V}$,

$$
\rho V=M_{1} n_{1}+M_{2} n_{2} .
$$


the Theory of Solutions.

If a mass $\mathbf{M}_{1} d n_{1}$ is added to the solution, we get

and

$$
\rho d v_{1}+\mathrm{V} d \rho=\mathrm{M}_{1} d n_{1}
$$

$$
\rho d v_{1}+\frac{\mathrm{M}_{1} n_{1}+\mathrm{M}_{2} n_{2}}{\rho} \frac{\partial \rho}{\partial e} \frac{\partial c}{\partial n_{1}} d n_{1}=\mathrm{M}_{1} d n_{1} .
$$

By a very simple calculation we get from this

$$
\frac{\mathrm{M}_{1}}{v_{1}}=\frac{\rho^{2}}{\rho+c(1+c) \frac{\partial \rho}{\partial c}} . . . .
$$

If the second component is treated in a similar manner,

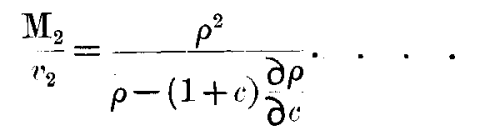

By help of equations (12a) and (12b) we can easily prove the following term :

$$
\frac{\partial v_{1}}{\partial r}+\epsilon \frac{M_{1}}{M_{2}} \frac{\partial v_{2}}{\partial}=0
$$

out of which immediately follow the equations

$$
k_{1}=v_{1} \text { and } k_{2}=v_{2} \text {. }
$$

According to a well known property of thermodynamic potential,

$$
\frac{\partial \omega}{\partial p}=\mathrm{V} .
$$

If we now introduce the values of $\omega$ (equation $4 b$ ) and $\mathrm{V}$, and obtain the partial derivatives with respect to $M_{1} n_{1}$ and $\mathrm{M}_{2} n_{2}$, we get

$$
\begin{aligned}
& \frac{\partial r_{1}}{\partial \rho}=\frac{\rho+c(1+c) \frac{\partial \rho}{\partial c}}{\rho^{2}}=\frac{v_{1}}{M_{1}}, \\
& \frac{\partial f_{2}}{\partial p}=\frac{\rho-(1+c) \frac{\partial \underline{\rho}}{\partial r}}{\rho^{2}}=\frac{r_{2}}{\mathrm{M}_{2}} .
\end{aligned}
$$

From these equations :

$$
\mathbb{M}_{1} \frac{\partial f_{1}}{\partial p}-\frac{M_{2}}{v_{2}} \frac{\partial f_{2}}{\partial p}=0 .
$$

The correctness of the equation (9) is thereby proved. 
When the terms found for $\frac{\mathrm{M}_{1}}{v_{1}}$ and $\frac{\mathrm{M}_{2}}{v_{2}}$ are introduced into equation (11 $\alpha$ ) a very simple calculation gives

$$
\rho \frac{\partial f_{1}(c, p, T)}{\partial c} \frac{\partial c}{\partial n}=-c(1+c) \frac{\partial \rho}{\partial c} . \text { K. . . }
$$

In this way it appears that we can obtain a determination of the concentration gradient by help of the thermodynamic condition of equilibrium. The result is therefore independent of every hypothesis as to the internal constitution of the solution. The formula cannot, however, be considered exact in a mathematical sense. In the course of the development we have taken it for granted that the fluid is incompressible, and that the molecule volumes do not change greatly with the concentration ; which, however, very nearly corresponds to real cases.

In our term for the concentration gradient, we have still to consider the function $f_{1}(c, p, T)$. Hitherto we have only mentioned that it is a function characteristic for both substances and closely connected with the external thermodynamic potential. In the work by Duhem above mentioned * the function is found for solations in which the concentration is small, i. e. for so-called Dilute Solutions.

Furthermore, van Laar $\dagger$, starting out from van der Waals's equation of state, has established a more general equation which is approximately applicable also to concentrated solutions.

Instead of $f_{1}(c, p, \mathrm{~T})$ we shall, however, introduce a quantity which is more spontaneously accessible to our conception-namely, the Osmotic Pressure. Not that the solution as such can, properly speaking, be ascribed to an osmotic pressure ; but let ns suppose that solution and solvent are in equilibrium on the two sides of a semipermeable membrane. The difference of hydrostatic pressures on the two sides of the membrane-the osmotic pressure $\pi$-is now connected with the function $f_{1}$ in the following simple manner :

$$
f_{1}(0, p, \mathrm{~T})-f_{1}(c, p, \mathrm{~T})=\frac{1}{\rho_{0}} \pi . \quad .
$$

$\rho_{0}$ is the density of the pure solvent since we have, throughout the development, calculated with mass units.

* Duhem, Mecanique Chimique, tome iii. livre vi. p. 44.

$\dagger$ See J. J. van Laar, Sechs Vorträge über das thermodynamische Potential, 1906, p. 85. 
$p$ is the pressure on the same side of the membrane as the solution. In order to apply these equations to our particular case, we must remember that we have to place the solution under this pressure. If equation (13) is differentiated with regard to $c$, we get

$$
\frac{\partial f_{1}^{\prime}(r, p, \mathrm{~T})}{\partial r}=\frac{1}{\rho_{0}}\left(\frac{\partial \pi}{\partial \iota^{\prime}}\right)_{p} .
$$

$\left(\begin{array}{l}\partial \pi \\ \partial c\end{array}\right)_{p}$ means the change of osmotic pressure per unit of concentration change, when the temperature and the pressure in the solution are kept constant. If this is introduced into equation (11 $b$ ), the result is

$$
\left(\begin{array}{l}
\partial \pi \\
\partial c
\end{array}\right)_{p} \frac{\partial c}{\partial \iota}=\frac{\rho_{0}}{\rho} c(1+c) \frac{\partial \rho}{\partial c} \mathrm{~K} . . . .
$$

It must be noticed that this equation holds good for all concentrations.

If the value of $\frac{\partial c}{\partial n}$ is introduced into equation (2):

$$
d l=-\frac{\rho_{0} r(1+c) \partial \rho}{\rho\left(\begin{array}{l}
\partial \pi \\
\partial r
\end{array}\right)_{p} \partial r} d \mathrm{U} . .
$$

The quantities $\left(\frac{\partial \pi}{\partial c}\right), \frac{\partial \rho}{\partial \rho}$, and $\rho$ are certain continuous functions of $c, p$, and $\mathrm{T}$ for the flnid mixture considered. As we have considered the fluid as incompressible, and as $\left(\frac{\partial \pi}{\partial c}\right)_{p}$ is approximately independent of the pressure, we may consider the three quantities as functions of $c$ and T. Term $(14 a)$ is then an exact differential ; and by integration, we get

$$
\int \frac{\rho\left(\frac{\partial \pi}{\partial \cdot}\right)}{\rho_{0^{\prime}}(1+c) \frac{\partial \rho}{\partial} \rho} \cdot d c=\mathrm{U} \cdot \quad \cdot \cdot \cdot
$$

The expression to the left, when integrated, gives a function of the concentration, which however contains the temperature as a parameter but not the pressure. By equation $(14 b)$ the concentration is determined as a function of the coordinates. 
We shall now consider some special cases :

(1) The solution is exposed to the influence of Gravity. Then we have

$$
\mathrm{U}=g h+\text { constant }
$$

$g$ is the acceleration of gravity, $h$ the height above a fixed horizontal plane. This being introduced into equation (14a),

$$
d c=-\frac{\rho_{0}}{\rho} \frac{(1+c)}{\left(\frac{\partial \pi}{\partial c}\right)_{p}} \frac{\partial \rho}{\partial c} g d h . \quad . .
$$

(2) The solution is placed in a vessel that turns round a vertical axis with constant velocity. It is supposed that the fluid follows the motion of the vessel. It is desired to find the potential $\mathrm{U}_{1}$ referred to a coordinate system, where the Z-axis coincides with the axis of rotation and the two other axes follow the motion. Then

and consequently

$$
d \mathrm{U}=y d z-\gamma^{2}(x d x+y d y),
$$

$$
d c=\rho_{\rho} \frac{c(1+c)}{\rho\left(\frac{\partial \pi}{\partial e}\right)_{p}} \frac{\partial \rho}{\partial e}\left[\gamma^{2}(x d x+y d y)-g d z\right] .
$$

The force-intensity at the considered point is expressed by the following term :

$$
\mathrm{K}=\sqrt{g^{2}+\gamma^{4}\left(x^{2}+y^{2}\right)}=\sqrt{g^{2}+\gamma^{4} \mathbf{R}^{2}} ;
$$

$\boldsymbol{\gamma}$ is the angular velocity and $\mathrm{R}$ the distance from the point to the axis of rotation.

As the concentration gradient is proportional to the force, we see that when $c$ and $\frac{\partial \rho}{\partial c}$ are not very small, we might make $\mathrm{R}$ and $\gamma$ sufficiently great to obtain a considerable change of concentration from place to place. In other words, we might expect to be able to separate the solutions for which $\frac{\partial \rho}{\partial c}$ has a considerable value, as milk is separated from cream in a separator. The separation will, however, never be complete. Besides it would be very difficult to carry out the experiment practically. In order that the separation might be made visible, the rotation would bave io be continued for a long time-since the rate of diffusion is very small-and the motion would have to be perfectly even. Furthermore, temperature differences would have to be avoided, as otherwise currents would arise in the fluid. 
Nevertheless Van Calcar and De Bruyn * have succeeded in showing that solutions really can be concentrated by centrifugalisation.

From the term for the concentration gradient we see, that-independent of the intensity of the force-it is equal to zero, when $\frac{\partial \rho}{\partial c}$ is equal to zero at the concentration present. In such a solution $d c$ will consequently be equal to zero, and the concentration will be the same throughout the solution. We shall suppose that we only have to do with the influence of gravity, and we shall notice the expression (11d) more minutely. If $\frac{\partial \rho}{\partial c}$ is positive, the density will increase with the concentration, and the concentration will decrease with the height. When $\frac{\partial \rho}{\partial c}$ is negative, the concentration increases upwards. From this it follows, as we have already pointed out at the beginning of this article, that in both cases the centre of gravity of the solution will be lowered by the action of the force.

For each solution we can consider the density as a function of the concentration, provided that the temperature and pressure are kept constant. This function can be represented as is well known, by putting $\rho$ proportional to the distance from the point to a fixed line $\mathrm{A}-\mathrm{B}$, and $c$ is represented by the ratios of the distances from the point to two lines perpendicular to $\mathrm{A}-\mathrm{B}$ (fig. 2). Each solution gives a curve. At a point where $\frac{\partial \rho}{\partial c}$ is equal to zero, the tangent of the curve is parallel to $A-B$ and the density has for this concentration an intermediate maximum (or minimum). If $\rho$ is a rational nonlinear function of $c$, it would always be possible to find one or more values of the concentration, for which $\frac{\partial \rho}{\partial c}$ is equal to zero. If, however, these values are to have a physical interpretation they must be positive and real, and also be within the domain of solubility. In fact we shall find, that to most solutions there will be no mixture proportion for which the concentration is constant throughout the solution. As the contraction of the solution is quite slight, we can take it for granted that the solution for which the gradient vanishes must consist of components whose densities differ very little from each other.

* Rec. Trav. Chem. Leiden, vol. xxiii. pp. 219-223 (1903). 
These proportions have been represented in the adjacent fig. 2, where four substances $S_{1}, S_{2}, S_{3}$, and $S_{4}$ are supposed to be dissolved in the same solvent. The density curves will

Fig. 2.

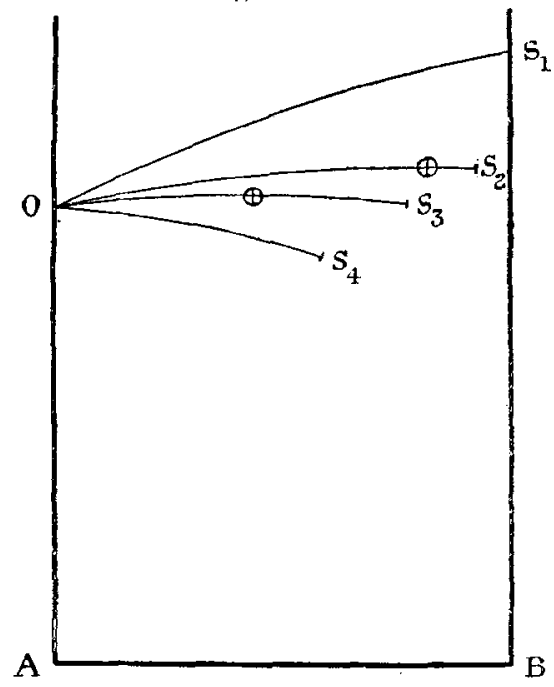

either rise always as $\mathrm{OS}_{1}$ or fall always as $\mathrm{OS}_{4}$. Only in the case of such curves which are nearly horizontal may we expect to find maximum points.

Finally we shall make a comparison between solutions and gases in this respect. If the absolute density is indicated by $\sigma$, we have in the case of gases

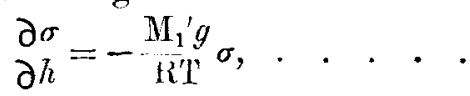

where $R$ is the gas constant for a molecular mass $M_{1}^{\prime}$ of the gas.

In order to get a term for the solution that might be compared with this, we must introduce another definition of the concentration, and mean now by the concentration the mass of the dissolved substances per volume unit of solution. If the concentration defined in this way is indicated by $\mathrm{C}$, we get the equations of transformation

$$
\begin{aligned}
& \mathrm{C}\left(\frac{1}{c}+1\right)=\rho \text {; } \\
& \left(\frac{\partial \pi}{\partial c^{\prime}}\right)_{p^{p}}=\left(\frac{\partial \pi}{\partial \mathrm{C}}\right)_{p^{3}} \cdot \mathrm{C}_{c^{\prime}} ;
\end{aligned}
$$

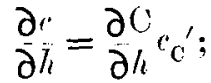


and remembering that $\mathrm{C}_{c}^{\prime}=\frac{1}{c_{\mathrm{o}}{ }^{\prime}}$, by introduction into $(11 d)$ :

$$
\left(\frac{\partial \pi}{\partial \mathrm{C}}\right)_{p} \frac{\partial \mathrm{C}}{\partial h}=-\frac{\rho_{0}}{\rho} c(1+c) \frac{\partial \rho}{\partial c} g .
$$

We will not transform the expression to the left. If the solution is not very concentrated, and the vapour-pressure of the second component may be disregarded in comparison with that of the solvent, we have approximately

$$
\left(\begin{array}{l}
\partial \pi \\
\partial \mathrm{C}
\end{array}\right)_{p}=\frac{1}{\mathrm{M}_{1}} \mathrm{RT} \text {. }
$$

$\mathrm{R}$ has the same value as before. If this is introduced,

$$
\frac{\partial(\mathrm{Y}}{\partial h}=-\frac{\rho_{0}}{\rho} \frac{\mathrm{M}_{1}}{\rho \mathrm{R} \mathrm{L}} c(\mathrm{I}+c) \frac{\partial \rho}{\partial c} g . . .
$$

A comparison between the equations (15 a) and (15b) immediately shows us that there is a fundamental difference between solutions and gases with regard to the change of concentration with the height. In the case of solutions $\frac{\partial \mathrm{C}}{\partial h}$ can, for instance, be equal to zero without the concentration itself being equal to zero, and this may just as well happen at the greatest concentrations. In the case of gases, however, the concentration-gradient will never be equal to zero, but will approach this value on the density decreasing ad infinitum. It might be said, however, that solutions and gases approach conformity, at unlimited dilution, since $\frac{\partial \mathrm{C}}{\partial h}$ as well as $\frac{\partial \sigma}{\partial h}$ then approach zero; but that similarity disappears in the case of concentrated solutions.

In the following table $\frac{\partial \mathrm{C}}{\partial h}$ has, according to equation (14b) been calculated for solutions of Cane Sugar and Potassium Hydrate at some concentrations*. The numbers refer to the absolute system of units. Then $\frac{\partial \mathrm{C}}{\partial h}$ is of the dimension

$$
\mid \text { Mass . Length }{ }^{-1}|=| \text { gr. } \mathrm{cm}^{-4} \text {. }
$$

* The density measurements are taken from Landolt and Börnstein. 
Mr. L. Vegard : Contributions to

\begin{tabular}{|c|c|c|c|c|}
\hline \multirow[b]{2}{*}{$c$} & \multicolumn{2}{|c|}{ Oane-Sugar Solution. } & \multicolumn{2}{|c|}{ KOH Solution. } \\
\hline & $\frac{\partial \rho}{\partial c}$ & $\frac{\partial C}{\partial h}$. & $\frac{\partial \rho}{\partial c}$ & $i \cdot \frac{\partial C}{\partial h}$. \\
\hline $1 / 19 \quad \ldots$ & 0.380 & $2 \cdot 87 \cdot 10^{-7}$ & $0 \cdot 720$ & $0 \cdot 87 \cdot 10^{-7}$ \\
\hline $1 / 9 \ldots \ldots$ & 0.337 & 5.57 & 0720 & $1 \cdot 87$ \\
\hline $3 / 17$ & 0.311 & $8 \cdot 49 \quad$, & $0 \cdot 650$ & 272 \\
\hline $1 / 4 \ldots \ldots$ & $0 \cdot 286$ & $1150 \quad$, & 0.700 & $4 \cdot 23$ \\
\hline $1 / 3 \ldots$ & $0 \cdot 260$ & $14 \cdot 55$ & 0.590 & $4 \cdot 85$ \\
\hline $1 \ldots$ & $0 \cdot 138$ & $31 \cdot 20$ & 0.320 & $9 \cdot 45$ \\
\hline
\end{tabular}

With regard to the calculation in the case of Potassium Hydrate, we must remark that such a solution is usually considered to be dissociated. The solution is then not binary in the strict sense of the word. If, however, the electrical action between the ions is very great compared with the influence of gravity, we can here suppose that the pairs of ions cannot be separated by the action of gravity, to any appreciable extent.

We can, however, not apply equation (15b) without further consideration. But equation (11e) still remains true, and we can write :-

$$
\left(\frac{\partial \pi}{\partial \mathrm{C}}\right)=i_{\mathrm{M}_{1}}^{1} \mathrm{R} \cdot \mathrm{T}
$$

where $i$ is the dissociation constant introduced by Van't Hoff, and which is generally dependent on the concentration.

Consequently

$$
{ }^{i} \frac{\partial \mathrm{C}}{\partial l}=-\frac{\rho_{0}}{\rho} \underset{\mathrm{R} \cdot \mathrm{T}}{\mathrm{M}_{1}} c(1+c) \frac{\partial \rho}{\partial c} g .
$$

The calculation has been carried out for the potassium hydrate solution according to this formula.

\section{$\$ 2$.}

On the Variation of the Osmotic Pressure with the Hydrostatic Pressure.

Osmotic pressure has already been mentioned in $\S 1$; as also what is to be understood by it. When defined in this manner, the osmotic pressure will not only depend on temperature and concentration, but also upon the pressure to which the fluid is exposed on one side of the membrane. In passing through the membrane from the solution into the solvent, the hydrostatic pressure will fall from $p$ to $p_{0}$, and 
the osmotic pressure is then defined by the equation,

at the same time

$$
\pi=p-p_{0} \text {. . . . . . }
$$

$$
\pi=\phi(c, p, \mathrm{~T}), . \quad . \quad . \quad . \quad .
$$

where $\phi$ is a single-valued, continuous function of $c, p$, and $\mathrm{T}^{*}$. When $p$ is introduced from equation (1), we get

This on solving for $\pi$ gives

$$
\pi=\phi\left(c, \pi+p_{0}, \mathrm{~T}\right) \text {. }
$$

$$
\pi=\phi_{0}\left(c, p_{0}, \mathbf{T}\right) \text {. }
$$

Consequently it is immaterial, whether we consider $\pi$ as a function of $p$ or of $p_{0}$.

We shall now try to find the influence of the external pressure upon the osmotic pressure. For this purpose we find the partial derivatives of $\pi$ with regard to $p$ or $p_{0}$-that is to say, the quantities $\frac{\partial \pi}{\partial p}$ and $\frac{\partial \pi}{\partial p_{0}}$. Let us suppose, that we have dissolved a ponderable substance in a ponderable Fig. 3.

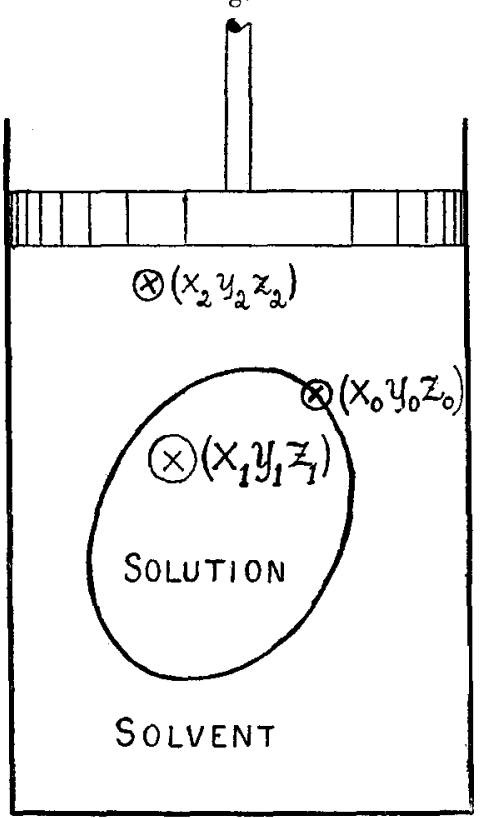

fluid. The solution is separated from the solvent by a semipermeable membrane, that forms a closed surface which we can suppose given by the equation

$$
\psi(x, y, z)=0 . \quad \text { (Fig. 3.) }
$$

* See Duhem, Mécanique Chimique, tome iii. livre vi. 
Let us furthermore suppose that the membrane is exceedingly thin, and that it can, at the same time, stand a great pressure ; conditions which cannot actually be realized in practice, but can be approximately, by fixing the membrane in a porcelain cell.

We consider the system after an ideal state of equilibrium has set in. As long as we are in one of the fluids, the general condition for mechanical equilibrium must be fulfilled. According to equation (1) $\S 1$, we get:-

(1) For the neighbourhood of a point $\left(x_{1}, y_{1}, z_{1}\right)$ of the solution :

$d p=-\rho\left(\frac{\partial \mathrm{U}\left(x_{1} y_{1} z_{1}\right)}{\partial x} d x+\frac{\partial \mathrm{U}\left(x_{1} y_{1} z_{1}\right)}{\partial y} d y+\frac{\partial \mathrm{U}\left(x_{1} y_{1} z_{1}\right)}{d z} d z\right) ;$

(2) For a point $\left(x_{2}, y_{2}, z_{2}\right)$ of the solvent:

$$
d p_{0}=-\rho_{0}\left(\frac{\partial U\left(x_{2} y_{2} z_{2}\right)}{\partial x} d x+\frac{\partial U\left(x_{2} y_{2} z_{2}\right)}{\partial y} d y+\frac{\partial U\left(x_{2} y_{2} z_{2}\right)}{\partial z} d z\right) .
$$

$\mathrm{U}$ means as before, the potential for the field to which the system is submitted. We suppose that $\mathrm{U}$, as well as its first partial derivatives with regard to $x, y, z$, are continuous functions in the space occupied by the system.

Now suppose the points $\left(x_{1} y_{1} z_{1}\right)$ and $\left(x_{2} y_{2} z_{2}\right)$ approach the point $\left(x_{0} y_{0} z_{0}\right)$, situated on the surface $\psi=0$; the total variation of osmotic pressure along the surface will be

$$
\left.\begin{array}{rl}
d \pi=d p-d p_{0}=-\left(\rho-\rho_{0}\right)\left(\frac{\partial \mathrm{U}\left(x_{0} y_{0} z_{0}\right)}{\partial x} d x\right. & +\frac{\partial \mathrm{U}\left(x_{0} y_{0} z_{0}\right)}{\partial y} d y \\
& \left.+\frac{\partial \mathrm{U}\left(v_{0} y_{0} z_{0}\right)}{\partial z} d z\right) \\
\frac{\partial \psi\left(x_{0} y_{0} z_{0}\right)}{\partial x} d x+\frac{\partial \psi\left(x_{0} y_{0} z_{0}\right)}{\partial y} d y+\frac{\partial \psi\left(x_{0} y_{0} \tilde{y}_{0}\right)}{d z} d z=0
\end{array}\right\}
$$

Besides, according to $(2 a)$ we have $\pi$ as a function of $\mathrm{T}, c$, and $p$. We suppose the temperature to be the same throughout the system, while $p$ and $c$ vary with the coordinates. Consequently we get

$$
d \pi=\left(\frac{\partial \pi}{\partial c}\right)_{p} d c+\frac{\partial \pi}{\partial p} d p .
$$

If the values for $d c$ and $d p$ are introduced from equations 
(1) and (2) $\$ 1$, we get

$$
\begin{aligned}
& d \pi=-\left[\left(\frac{\partial \pi}{\partial \rho}\right)_{p} \frac{\partial r}{\partial n} \frac{1}{\mathrm{~K}}+\frac{\partial \pi}{\partial p} \rho\right]\left(\frac{\partial \mathrm{U}\left(x_{\left.0, y_{0} z_{0}\right)}\right)}{\partial x} d x+\frac{\partial \mathrm{U}\left(x_{0} y_{0} \tilde{v}_{0}\right)}{\partial y} d y\right. \\
& \left.+\frac{\partial U\left(x_{0} y_{0} z_{n}\right)}{\partial z} d z\right) \\
& \frac{\partial \psi\left(x_{0} y_{0} z_{0}\right)}{\partial u} d x+\frac{\partial \psi\left(x_{0} y_{0} z_{1}\right)}{\partial y} d y+\frac{\partial \psi\left(x_{0} y_{0} z_{0}\right)}{\partial z} d z=0 . \quad j
\end{aligned}
$$

As both expressions for the total differential of $\pi$ along the membrane must be identical, we get as the necessary condition for it :

$$
\rho-f_{0}=\left(\frac{\partial \pi}{\partial c}\right)_{p} \partial \frac{\partial c}{\partial \mu} K^{-}+\rho \frac{\partial \pi}{\partial p} \cdot . .
$$

If we choose $p_{0}$ instead of $p$ as variable, we get in a similar manner

$$
\rho-\rho_{0}=\left(\frac{\partial \pi}{\partial c}\right)_{p_{0}} \frac{\partial c}{\partial n} \frac{1}{\mathrm{~K}}+\rho_{0} \frac{\partial \pi}{\partial p_{0}} \quad . \quad .
$$

By means of equations $(4 a)$ and $(4 b)$ there has been developed a simple connexion between the concentration gradient and the dependence of the Osmotic Pressure npon the External Pressure, a connexion which makes it possible to calculate one of these quantities when the other is known. In the development of this formula nothing has been assumed that restricts the general applicability of the solution found; the fluid may also even be compressible. The formula will hold good, provided always that the fluid fulfils the equilibrium condition (1) $\S 1$, characteristic of an ideal fluid.

We will now proceed to apply the formula for the calculation of $\frac{\partial \pi}{\partial p}$, as we have already found a value for $\frac{\partial c}{\partial n}$ in the first section. By the aid of equation (11c) $\S 1$ we get

$$
\underline{\frac{\partial \pi}{\partial p}}=\frac{\rho-\rho_{0}}{\rho}-\frac{\rho_{0}}{\rho^{2}} c(1+c) \frac{\partial \rho}{\partial c} . . .
$$

The term that we in this way have found for the dependence of Usmotic Pressure upon External Pressure, is now fully in accordance with the equations found by Planck and Duhem from another point of yiew.

Phil. Mag. S. 6. Vol. 13. No. 77. May 1907. 
Planck * has expressed the law in the following terms:

$$
v_{0} d_{p_{0}}=v d p \text {. }
$$

$v_{0}$ is the volume of $1 \mathrm{gr}$. solvent, $v$ is the increase of volume suffered by a very great quantity of the solution, when $1 \mathrm{gr}$. of solvent is added to it. If equation (1) is taken into consideration, we get

$$
\frac{\partial \pi}{\partial l^{\prime}}=1-\frac{v}{v_{0}}
$$

In order to find the proportion $\frac{v}{v_{0}}$ we will take a limited instead of an unlimited quantity, and add to it an exceedingly small quantity of the solvent. We have as before:

$$
\mathrm{V} \rho=\mathrm{M}_{1} n_{1}+\mathrm{M}_{2} n_{2} \text {. }
$$

If we add a quantity $\mathrm{M}_{1} d n_{1}=\rho_{0} d v_{0}$ of the first component we get:

$$
d v=\frac{v}{v_{0}}=\frac{\rho_{0}}{\rho}\left(1+\frac{1}{\rho} c(1+c) \frac{\partial \rho}{\partial c}\right) . . .
$$

From equations (6) and (7) we almost immediately get equation (5).

Duhem $\dagger$ has developed the following formula:

$$
\frac{\mathrm{P}_{1}-\mathrm{P}}{\Pi_{1}^{\prime}-\mathbf{I}}=\frac{\mathrm{U}_{1}(\mathrm{~T})-v(c, \mathrm{~T})+c(1+c) \frac{\partial v(c, \mathrm{~T})}{\partial c}}{v(c, \mathrm{~T})-c(\mathbf{1}+c) \frac{\partial v(c, \mathrm{~T})}{\partial c}} .
$$

The term to the left corresponds fully to what we have called $\frac{\partial \pi}{\partial p}$. Furthermore, we have :

$$
\mathrm{U}_{1}(\mathrm{~L})=\frac{1}{\rho_{0}} \text { and } v(c, \mathrm{~T})=\frac{1}{\rho} \text {. }
$$

If this is introduced, we get:

$$
\frac{\partial \pi}{\partial p}=\frac{\frac{\rho}{\rho_{0}}-\left(1+\frac{1}{\rho} c(1+c) \frac{\partial \rho}{\partial c}\right)}{1+\frac{1}{\rho} c(1+c) \frac{\partial \rho}{\partial c}},
$$

* Planck, Zeitschrift für physik. Chemie, xliii. p 584.

+ Mécanique Chimique, tome iv. livre vi. p. 65 . 
and with the help of equation (7):

$$
\frac{\partial \pi}{\partial \eta}=\frac{1-v_{0}}{\frac{r}{r_{0}}} .
$$

But $\left(1-\frac{v}{v_{0}}\right)$ is a very small quantity in comparison with the unit. We can therefore, when quantities of second and higher order are left out of consideration, immediately introduce one in the dominator and get the formula of Planck.

As already mentioned the formula (4a) holds good independently of the compressibility as also of the molecular volume's variation with the concentration.

In Planck's formula the contraction is also taken into consideration. From equations $(4 a),(6)$, and (7), we can now reversibly deduce the expression found in $\$ 1$ for the concentration gradient.

The dependence of Osmotic Pressure upon External Pressure has also been theoretically treated by O. E. Schiötz* . $\mathrm{He}$ comes to the result, that the change of pressure on the two sides of the membrane is connected by the following equation:

and consequently

$$
\rho d p=\rho_{0} d p_{0}
$$

$$
\frac{\partial \pi}{\partial p}=\frac{\rho-\rho_{0}}{\rho}
$$

This value for $\frac{\partial \pi}{\partial p}$ is obviously appreciably different from the one found above. The reason for the discrepancy must be sought in the fact, that Schiötz in developing this formula, has not taken under consideration the fact that the concentration varies with the height. 'This, however, must be the case, as we have seen. From our formula it appears that when the solution fulfils the condition $\frac{\partial c}{\partial n}=0$, the formula developed by Schiötz will hold good for this special solution.

We shall finally make a few comments concerning the variation of Osmotic Pressure with Concentration. In order to examine the influence of the concentration, we can suppose

* O. E. Schiötz, "Ueber die Abhangigkeit des osmot. Druckes und der Dampfspannung von dem Drucke" (Boltzmann-Festschrift, 1904, p. 618). 
that it changes while the temperature is maintained constant; but still the change of concentration can occur under two different circumstances.

Either, the pressure can be kept constant in the solution; then we get the relation indicated by $\left(\frac{\partial \pi}{\partial c}\right)_{p}$. Or we can keep the pressure on the solvent constant-in practice the more general case-and we get $\left(\frac{\partial \pi}{\partial c}\right)_{p_{0}}$.

We can now prove that these two quantities must generally differ from each other. If equations (4a) and (4b) are subtracted,

$$
\left[\left(\frac{\partial \pi}{\partial c}\right)_{p}-\left(\frac{\partial \pi}{\partial c}\right)_{p_{0}}\right]_{\mathrm{K}}^{1} \frac{\partial c}{\partial n}=\rho_{0} \frac{\partial \pi}{\partial p_{0}}-\rho \frac{\partial \pi}{\partial p} .
$$

Let us now suppose that $\frac{\partial c}{\partial n}$ has a finite value, which is generally the case. The necessary and sufficient condition for $\left(\frac{\partial \pi}{\partial c}\right)_{p}$ being equal to $\left(\frac{\partial \pi}{\partial c}\right)_{p_{c}}$ is that

$$
\rho_{0} \frac{\partial \pi}{\partial x_{0}}=\rho \frac{\partial \pi}{\partial p} \cdot \quad . \quad . \quad . \quad . \quad . \quad .
$$

Generally, however, this equation is not fulfilled. To prove this, let us suppose that we increase the pressure on the two sides by just so much, that equilibrium is not disturbed.

Fig. 4.

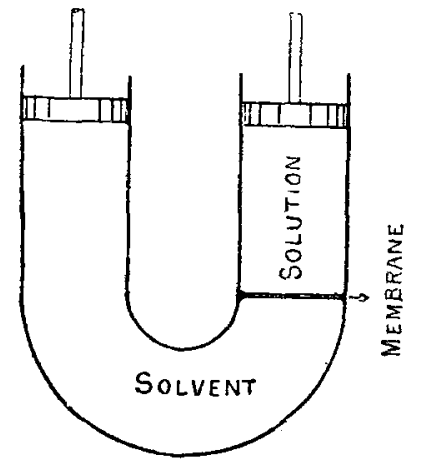

We increase the pressure without moving along the membrane. Such a change of pressure can be carried out, for instance, by means of the arrangement represented in fig. 4. 
On this supposition

and consequently,

$$
d \pi=d p-d p_{0}=\frac{\partial \pi}{\partial p} d p-\frac{\partial \pi}{\partial p_{0}} d p_{0},
$$

$$
\frac{\partial \pi}{\partial p_{0}}=\frac{\partial \pi}{\partial p}\left(1+\frac{\partial \pi}{\partial p_{0}}\right)
$$

In order that equation ( 8 ) also be fulfilled, we must have

$$
\stackrel{\rho}{\rho} \frac{\partial \pi}{\rho_{0}} \partial \eta=\frac{\partial \pi}{\partial p}\left(1+\frac{\rho}{\rho_{0}} \frac{\partial \pi}{\partial \nu}\right)
$$

That this equation be fulfilled, we must either have

$$
\text { or } \quad \begin{aligned}
& \frac{\partial \pi}{\partial l^{\prime}}=0 \\
& \frac{\partial \pi}{\partial p}=\begin{array}{c}
\rho-\rho_{0} \\
\rho
\end{array} .
\end{aligned}
$$

The first of these equations implies that the osmotic pressure is independent of external pressure, which will not be the case generally.

The second equation can according to $(4 a)$ only be fulfilled when $\frac{\partial c}{\partial n}$ is equal to zero; but this is in contradiction to our supposition. If, however, we had to do with such a special case, we should get

$$
\left(\frac{\partial \pi}{\partial c}\right)_{\nu}-\left(\frac{\partial \pi}{\partial c}\right)_{\nu_{0}}=\frac{0}{U} .
$$

This is apparently an indeterminate expression, which is, however, determined by the limiting value

$$
\text { K. Lim. }\left(\frac{\rho_{0} \frac{\partial \pi}{\partial p_{0}}-\rho \frac{\partial \pi}{\partial p}}{\partial c}\right)_{\frac{\partial c}{\partial n}=0}
$$

We thus come to the result, so very important to the theory of Osmotic Pressure, that the variation of this pressure with the concentration will be different, according as the pressure during the variation is kept constant on the solution or on the solvent. 